\title{
High mobility group box 1 and kidney diseases (Review)
}

\author{
PING ZHU ${ }^{1}$, LI XIE ${ }^{2}$, HUA-SHENG DING ${ }^{3}$, QUAN GONG $^{4}$, JIAN YANG $^{3}$ and LIN YANG ${ }^{1}$ \\ Departments of ${ }^{1}$ Nephrology, ${ }^{2}$ General Surgery and ${ }^{3}$ Cardiology, The First College of Clinical Medical Science, \\ China Three Gorges University, Yichang; ${ }^{4}$ Department of Immunology, Tongji Medical College, \\ Huazhong University of Science and Technology, Wuhan, P.R. China
}

Received November 27, 2012; Accepted January 18, 2013

DOI: $10.3892 / \mathrm{ijmm} .2013 .1286$

\begin{abstract}
High mobility group box 1 (HMGB1), a nonhistone DNA-binding protein, regulates nucleosome function and transcription in the nuclei of all metazoans and plants. However, extracellular HMGB1, which is actively or passively released under different conditions, can act as a key inflammatory mediator through MyD88/mitogen-activated protein kinase signaling by binding to its receptors including the receptor for advanced glycation end products or Toll-like receptors. A growing body of evidence indicates that HMGB1 plays an important role in kidney diseases, such as glomerulonephritis, lupus nephritis, antineutrophilic cytoplasmatic antibody-associated vaculitis, diabetic nephropathy, renal allograft rejection and acute kidney injury. In this review, we focus on the biology of HMGB1 and the association of HMGB1 with kidney diseases.
\end{abstract}

\section{Contents}

\author{
1. Introduction \\ 2. Structure of HMGB1 \\ 3. Function of intra-nuclear HMGB1 \\ 4. Release of HMGB1 \\ 5. Ligands and signaling of HMGB1 \\ 6. HMGB1 and kidney diseases \\ 7. Conclusion
}

\section{Introduction}

High mobility group box 1 (HMGB1) was originally described 30 years ago as a nonhistone DNA-binding protein with high-electrophoretic mobility (1). It is present in the nucleus of almost all metazoans and plants, where it exerts structural

Correspondence to: Professor Ping Zhu, Department of Nephrology, The First College of Clinical Medical Science, China Three Gorges University, Yichang 443003, P.R. China

E-mail: topgan2000@163.com

Key words: high mobility group box 1, receptor for advanced glycation end products, Toll-like receptors, kidney diseases and transcriptional activities (2-4). In addition to its role in the nucleus, HMGB1 has recently emerged as an extracellular signaling factor with key roles in cell differentiation, proliferation and disease pathogenesis (5).

In this review, we focus on the involvement of HMGB1 in the pathogenesis of kidney diseases, as well as renal allograft rejection after renal transplantation. An understanding of these discoveries may shed new insight into the possibilities for developing novel therapeutic strategies to mitigate or prevent kidney diseases.

\section{Structure of HMGB1}

The HMGB1 protein, a member of the high mobility group nuclear protein family, is one of the most evolutionarily conserved proteins and shares $99 \%$ identity in the amino acid sequence between rodents and humans (1). The human HMGB1 gene is located on chromosome 13q12 (6), and six polymorphic loci throughout the gene locus have recently been identified (7).

HMGB1 has a highly dipolar structure consisting of 215 residues organized into two basic DNA-binding domains, referred to as the A and B box as well as a negatively charged C-acidic tail (Fig. 1) (8). Each HMGB1 A or B box is approximately 75-80 amino acids in length (9) and is formed by two short and one long $\alpha$-helix that upon folding produce an L- or V-shaped three-dimensional domain structure $(10,11)$. Research suggests that the B box possesses the pro-inflammatory properties of HMGB1 including cytokine release, and the A box instead competes with HMGB1 for binding sites leading to attenuation of the inflammatory cascade $(12,13)$.

\section{Function of intra-nuclear HMGB1}

As the HMGB1 protein is essential for life, HMGB1-knockout mice die shortly after birth (14). HMGB1 is fairly ubiquitous in mammals and is almost always present in the nucleus (15) where HMGB1 plays an important role in binding without sequence specificity to the minor groove of DNA and induces bends in the helical structure, which regulate physical interactions between DNA and transcription factors, including p53, homeobox proteins, glucocorticoid receptor, recombinationactivating gene 1/2 (RAG1/2) proteins and steroid hormone receptors $(16,17)$. Even though HMGB1 is not essential for the overall organization of chromatin in the nucleus, it is critical 


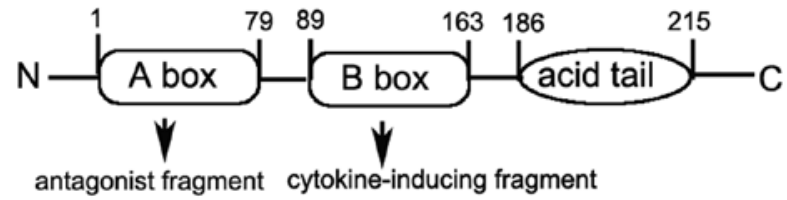

Figure 1. Structure of HMGB1. The full-length HMGB1 is subdivided into two homogeneous domains (A and $\mathrm{B}$ box) and an acidic tail. The A box (AA1-79) is a specific antagonist, while the B box (AA89-163) is the RAGEbinding domain that induces cytokines to promote inflammation.

for proper transcriptional control by specific transcription factors $(14,18)$.

\section{Release of HMGB1}

In addition to its roles in regulating nucleosome function and transcription, HMGB1 has recently emerged as an extracellularly release mediator of inflammation, although it lacks a classical secretion signal. There are two modes of HMGB1 release: one active and one passive. HMGB1 not only is passively released from damaged cells under conditions of injury, but can also be actively secreted from activated immune cells in response to inflammatory stimuli $(18,19)$.

How is HMGB1 released? A growing body of evidence indicates that inflammasomes play an important role in the release of HMGB1. Recently, NLRP3 inflammasome activation was shown to be essential for HMGB1 release from LPS-primed macrophages treated with ATP or exposed to nigericin (20). Similarly, the extracellular release of HMGB1 from S. typhimurium-infected macrophages was found to rely on activation of caspase-1 by the NLRC4 inflammasome (20). However, the exact mechanism remains unclear (Fig. 2).

\section{Ligands and signaling of HMGB1}

To date, several important surface receptors have been implicated in HMGB1 signaling, including the receptor for advanced glycation end products (RAGE), Toll-like receptor (TLR)2, TLR4 and syndecan $(21,22)$. Binding to these receptors results in the activation of nuclear factor (NF)- $\mathrm{BB}$, which induces the upregulation of pro-inflammatory cytokines, thereby promoting inflammation.

RAGE which is expressed on monocytes, macrophages, neurons and endothelial cells, as well as on a variety of tumor cells is thought to be one of the primary receptors for HMGB1 $(23,24)$. Interaction of HMGB1 with RAGE can activate two major signaling pathways, one encompassing $\mathrm{CDC} 42 / \mathrm{Rac}$ and the other involving diverse mitogen-activated protein kinases (MAPKs) that finally leads to cytoskeletal changes and NF- $\mathrm{KB}$ activation, respectively $(25,26)$.

In addition to RAGE, TLR 2 and 4 which are expressed on antigen-presenting cells (APCs) were found to directly interact with HMGB1 as determined by fluorescence resonance energy transfer (FRET) and immunoprecipitation (27). Numerous studies have demonstrated that HMGB1 signaling through TLR2 and TLR4 is mediated by the Rac1/phosphoinositide3-kinase (PI3K)/CDC42 pathway and MyD88-dependent $\mathrm{NF}-\kappa \mathrm{B}$ activation pathway, respectively $(21,28,29)$.
Recently, numerous studies have demonstrated that HMGB1 acts as a CpG-ODN-binding protein, by which it interacts and preassociates with TLR9 in the endoplasmic reticulum-Golgi intermediate compartment (ERGIC), and as a result, forms a complex within specialized vesicles (30) which is considered as an accelerator of TLR9 response with CpG-DNA (31). HMGB1 accelerates the delivery of $\mathrm{CpG}-\mathrm{ODNs}$ to its receptor, leading to a TLR9-dependent augmentation of interleukin (IL)-6, IL-12 and tumor necrosis factor (TNF)- $\alpha$ secretion (30).

More recently, apart from RAGE and TLRs, HMGB1 can interact with a wide range of proteins with a phage display approach (10). Yet, further studies are required to determine the exact function of HMGB1 interaction with these proteins.

\section{HMGB1 and kidney diseases}

HMGB1 and glomerulonephritis. Granulomatous nephritis is triggered by a diverse group of factors and results in renal failure. Granulomatous inflammation is one of the most significant pathogenetic mechanisms in nephritis. Recently, research revealed that the HMGB1 level in urine and serum was elevated in crystal-induced granulomatous nephritis caused by an adenine-rich diet, and HMGB1 induced monocyte chemoattractant protein-1 (MCP-1) secretion through the MAPK and PI3K pathways. The authors concluded that HMGB1 is a new mediator involved in crystal-induced nephritis that amplifies granulomatous inflammation through a cycle in which MCP-1 attracts activated macrophages, resulting in excessive and sustained HMGB1 release. Thus, HMGB1 may be a novel target for the prevention or treatment of granulomatous nephritis (32).

\section{HMGB1 and secondary kidney diseases}

HMGB1 and lupus nephritis. Lupus nephritis is common in systemic lupus erythematosus (SLE) patients and manifests mainly by proteinuria, hematuria, and, less commonly, severe renal failure. The mechanism of lupus nephritis is not fully clear. Yet, accumulating data suggest that HMGB1 may play an important role in lupus nephritis.

Iwata et al (33) revealed that HMGB1 secreted by dendritic cells via p38 MAPK activation participates in autoimmunity in MRL-Fas(lpr) mice (a lupus-prone mouse model). These results suggest that HMGB1 is involved in the progression of autoimmune kidney diseases in MRL-Fas(lpr) mice (33).

Research reveals that high expression of HMGB1 (in blood or renal biopsies) is positively correlated with MCP-1 expression and may contribute to the pathogenesis of lupus nephritis $(34,35)$. Recently, Feng et al (36) demonstrated that HMGB1 mRNA and protein levels were increased in the glomeruli of lupus nephritis patients and BXSB mice. Their findings indicate that HMGB1 mediates interferon (IFN)- $\gamma$-induced cell proliferation in mouse mesangial cells through regulation of the cyclin D1/CDK4/p16 pathway and promotion of cell cycle transition from G1 to $\mathrm{S}$ stage in lupus nephritis (36). Therefore, the above findings clearly indicate a key role of HMGB1 in lupus nephritis, and inhibition of HMGB1 or MCP-1 may be a novel treatment strategy for lupus nephritis $(34,37)$.

HMGB1 and antineutrophilic cytoplasmatic antibody (ACNA)-associated vasculitis (AAV). Cytokines, such as 


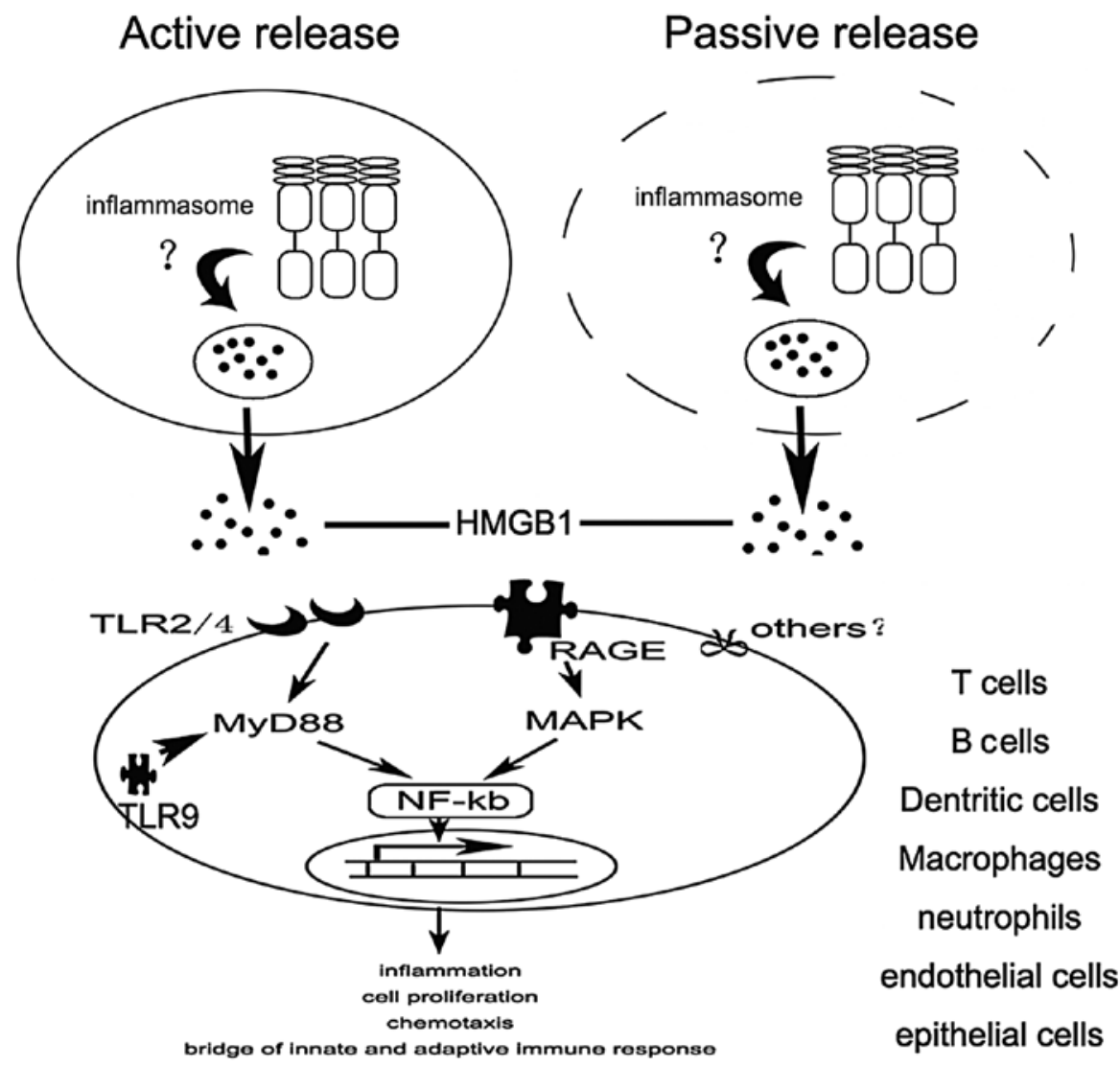

Figure 2. A diagram illustrating HMGB1 release, ligands and signaling. HMGB1 is released from cells either by an active (along with pro-inflammatory stimuli) or passive (damaged and infected cells undergoing necrosis) manner through an inflammasome mechanism which remains unelucidated. Released HMGB1 protein binds to its receptors (TLR2, 4, 9 and RAGE) on effector cells to induce inflammation, cell proliferation, chemotaxis and bridge the innate and adaptive immune response.

TNF and IL-6, are important in ACNA-AAV. HMGB1 can induce the release of TNF and IL- 6 by APCs. Recently, Bruchfeld et al (38) concluded that the level of HMGB1 is increased in AVV with renal manifestations. This suggests that HMGB1 plays an important role in AVV.

HMGB1 and diabetic nephropathy. Research has shown that the expression of HSP70 and HMGB1, endogenous ligands of TLRs, is significantly upregulated in the kidneys of diabetic rats. These findings suggest that release of hyperglycemia-induced HMGB1 may induce renal injury in diabetic rats, and that the pathogenic role of HMGB1 may be dependent on RAGE or TLR4 and through activation of $\mathrm{NF}-\kappa \mathrm{B}$ and may promote tubulointerstitial inflammation in diabetic nephropathy $(39,40)$. In contrast, other results found that the level of HMGB1 in serum was decreased in patients with diabetic nephropathy (41). Further extensive study is required to explore the role of HMGB1 in diabetic nephropathy.

HMGB1 and autosomal dominant polycystic kidney disease. Autosomal dominant polycystic kidney disease (ADPKD) is the most common monogenic kidney disease and the fourth leading cause of end-stage kidney disease in adults worldwide (42). Recently, two research groups reported that the serum level of HMGB1 was increased in ADPKD patients $(43,44)$ which suggests that HMGB1 may play a role in ADPKD.
HMGB1 and acute kidney injury. In 1999, HMGB1 was implicated as a late mediator of lethal systemic inflammation in sepsis (18). As acute kidney injury is a severe complication of sepsis, there is evidence to indicate that HMGB1 plays a significant role in sepsis-mediated acute kidney injury (45).

Wang et al (46) found that HMGB1 expression was markedly increased in renal tissue and in acute kidney injury in rats with delayed resuscitation after thermal injury. Chen et al (47) found that HMGB1 released by injured renal cells in renal ischemia and reperfusion injury (RIRI) can induce TLR4 (+/+) leukocytes producing IL- 6 by binding to its receptor TLR4. This underscores the importance of HMGB1/TLR4 signaling in the pathogenesis of ischemic acute kidney injury.

In addition, Chung et al (48) found that HMGB1 expression was increased in the kidney $6 \mathrm{~h}$ after reperfusion and was decreased gradually 1, 3 and 5 days following reperfusion. Moreover, Wu et al (49) demonstrated that mice treated with anti-HMGB1 antibody had significantly less tubulointerstitial infiltration by neutrophils (day 1) and macrophages (day 5) and markedly reduced apoptosis of tubular epithelial cells. Furthermore, anti-HMGB1 antibody-treated IRI kidneys had significantly lower levels of IL-6, TNF and MCP-1 mRNA, which are downstream of HMGB1. Conversely, administration of rHMGB1 after reperfusion exacerbated kidney IRI in wildtype mice. They conclude that HMGB1 contributes to kidney 
Table I. The main role of HMGB1 in kidney diseases.

Kidney diseases

Role of HMGB1

HMGB1 and glomerulonephritis

HMGB1 and secondary kidney diseases

HMGB1 and lupus nephritis

HMGB1 and ACNA-associated vasculitis

HMGB1 and diabetic nephropathy

HMGB1 and dominant polycystic kidney disease

HMGB1 and acute kidney injury

Acute kidney injury of sepsis

Acute kidney injury of ischemia and reperfusion injury

HMGB1 and chronic kidney diseases

HMGB1 and renal cell carcinoma

HMGB1 and chronic allograft dysfunction
Promotes inflammation and induces MCP-1 secretion

Promotes inflammation and induces MCP-1 secretion; mediates IFN- $\gamma$-induced cell proliferation

Mediates the immune response

Activation of NF-kB; chemotactic; promotes

tubulointerstitial inflammation

Promotes inflammatory injury

Mediates inflammation; increases influx of neutrophils Activation of macrophages; induces cytokine release; reduces apoptosis of tubular epithelial cells

Promotes immune response; enhances ADMA levels; immune-mediated EMT

Activation of ERK1/2

Promotes inflammation through MyD88 and TRIF signaling

HMGB1, high mobility group box 1; MCP-1, monocyte chemoattractant protein-1; ADMA, accumulation of asymmetric dimethylarginine; EMT, epithelial-mesenchymal transition; IFN- $\gamma$, interferon; NF- $\mathrm{KB}$, nuclear factor.

ischemia reperfusion injury (50). These findings demonstrate that HMGB1 plays an important role in acute kidney injury.

HMGB1 and chronic kidney disease. Chronic kidney disease is a multifactorial disorder occurring in the context of chronic conditions of co-morbidity. Research has indicated that chronic kidney disease is an immune inflammatory condition (51). There is accumulating evidence linking HMGB1 and chronic kidney disease.

Bruchfeld et al (52) revealed that HMGB1 is significantly elevated in patients with chronic kidney disease and correlates with GFR as well as markers of inflammation and malnutrition. Another study found that HMGB1 was expressed in the serum of patients with renal diseases who underwent renal biopsies, particularly patients who suffered from vasculitis including ANCA-GN, Henoch-Schonlein purpura nephritis, and IgAN with glomerular crescents (53). Moreover, Leelahavanichkul et al (54) suggested that HMGB1 is an important common mediator for both chronic kidney disease and sepsis.

Nakamura et al (55) demonstrated that HMGB1 enhances the accumulation of asymmetric dimethylarginine (ADMA) levels, suggesting the active involvement of the AGE/HMGB1RAGE-ADMA axis in nondiabetic chronic kidney disease patients and that inhibition of HMGB1/RAGE may be a strategy for the treatment of chronic kidney disease (56).

In addition, research suggests that HMGB1 is a key mediator of immune-mediated epithelial-mesenchymal transition (EMT) of proximal tubular epithelial cells and a potentially important signaling molecule in the development of renal fibrosis (57).
HMGB1 and clear cell renal cell carcinoma. Renal cell carcinoma is the most common cancer of the kidney. The main histological subtypes are clear cell (75\%), papillary (15\%) and chromophobe renal cell carcinoma (5\%) (58). Recently, Lin et al (59) demonstrated that HMGB1 promotes the development and progression of clear cell renal cell carcinoma via ERK1/2 activation, which is partially mediated by RAGE. This suggests that HMGB1 is involved in clear cell renal cell carcinoma.

HMGB1 and chronic allograft dysfunction. Chronic allograft dysfunction, a leading cause of chronic allograft failure among kidney transplant recipients, is a multifactorial process associated with progressive interstitial fibrosis and tubular atrophy (60). Recently, Wang et al (61) found that the level of HMGB1/TLR4 was increased in chronic renal transplantation patients. Their findings indicate the MyD88 and TRIF signaling plays an important role in graft-infiltrating mononuclear cells in the pathophysiology of chronic allograft dysfunction (61). These findings suggest that HMGB1 may be an effective target for the prevention and treatment of chronic allograft dysfunction.

\section{Conclusion}

In conclusion, the robust associations between HMGB1 and kidney diseases have been reviewed in this article (Table I). Although the mechanisms promoting the release of HMGB1 and the signaling pathways it activates require further elucidation, evidence suggests its potential as a therapeutic target/ agent in various kidney diseases. Considering its notable 
role in kidney diseases, a therapeutic approach involving the HMGB1-mediated signaling pathway may constitute a new strategy for the treatment of kidney diseases. Future research may aid in determining the feasibility of such an approach.

\section{References}

1. Goodwin GH, Sanders C and Johns EW: A new group of chromatin-associated proteins with a high content of acidic and basic amino acids. Eur J Biochem 38: 14-19, 1973.

2. Bustin M, Lehn D and Landsman D: Structural features of the HMG chromosomal proteins and their genes. Biochim Biophys Acta 1049: 231-243, 1990.

3. Bonaldi T, Längst G, Strohner R, Becker PB and Bianchi ME: The DNA chaperone HMGB1 facilitates ACF/CHRACdependent nucleosome sliding. EMBO J 21: 6865-6873, 2002.

4. Travers AA: Priming the nucleosome: a role for HMGB proteins? EMBO Rep 4: 131-136, 2003.

5. Hock R, Furusawa T, Ueda T and Bustin M: HMG chromosomal proteins in development and disease. Trends Cell Biol 17: 72-79, 2007.

6. Ferrari S, Finelli P, Rocchi M and Bianchi M: The active gene that encodes human high mobility group 1 protein (HMG1) contains introns and maps to chromosome 13 . Genomics 35 367-371, 1996.

7. Kornblit B, Munthe-Fog L, Petersen S, Madsen H, Vindeløv L and Garred P: The genetic variation of the human HMGB1 gene. Tissue Antigens 70: 151-156, 2007.

8. Landsman D and Bustin M: A signature for the HMG-1 box DNA-binding proteins. Bioessays 15: 539-546, 1993

9. Han J, Zhong J, Wei W, et al: Extracellular high-mobility group box 1 acts as an innate immune mediator to enhance autoimmune progression and diabetes onset in NOD mice. Diabetes 57: 2118-2127, 2008.

10. Dintilhac A and Bernués J: HMGB1 interacts with many apparently unrelated proteins by recognizing short amino acid sequences. J Biol Chem 277: 7021-7028, 2002.

11. Weir H, Kraulis P, Hill C, Raine A, Laue E and Thomas J: Structure of the HMG box motif in the B-domain of HMG1. EMBO J 12: 1311-1319, 1993.

12. Messmer D, Yang H, Telusma G, et al: High mobility group box protein 1: an endogenous signal for dendritic cell maturation and Th1 polarization. J Immunol 173: 307-313, 2004.

13. Yang H, Ochani M, Li J, et al: Reversing established sepsis with antagonists of endogenous high-mobility group box 1 . Proc Nat Acad Sci USA 101: 296-301, 2004.

14. Calogero S, Grassi F, Aguzzi A, et al: The lack of chromosomal protein Hmg1 does not disrupt cell growth but causes lethal hypoglycaemia in newborn mice. Nat Genet 22: 276-280, 1999.

15. Müller S, Scaffidi P, Degryse B, et al: New EMBO member's review: the double life of HMGB1 chromatin protein: architectural factor and extracellular signal. EMBO J 20: 4337-4340, 2001.

16. Ulloa L, Batliwalla FM, Andersson U, Gregersen PK and Tracey KJ: High mobility group box chromosomal protein 1 as a nuclear protein, cytokine, and potential therapeutic target in arthritis. Arthritis Rheum 48: 876-881, 2003.

17. Brickman JM, Adam $M$ and Ptashne $M$ : Interactions between an HMG-1 protein and members of the Rel family. Proc Natl Acad Sci USA 96: 10679-10683, 1999.

18. Wang H, Bloom O, Zhang M, et al: HMG-1 as a late mediator of endotoxin lethality in mice. Science 285: 248-251, 1999.

19. Bianchi ME and Manfredi AA: High-mobility group box 1 (HMGB1) protein at the crossroads between innate and adaptive immunity. Immunol Rev 220: 35-46, 2007.

20. Lamkanfi M, Sarkar A, Vande Walle L, et al: Inflammasomedependent release of the alarmin HMGB1 in endotoxemia. J Immunol 185: 4385-4392, 2010.

21. Park JS, Svetkauskaite D, He Q, et al: Involvement of toll-like receptors 2 and 4 in cellular activation by high mobility group box 1 protein. J Biol Chem 279: 7370-7377, 2004.

22. Kokkola R, Andersson A, Mullins G, et al: RAGE is the major receptor for the proinflammatory activity of HMGB1 in rodent macrophages. Scand J Immunol 61: 1-9, 2005.

23. Hori O, Brett J, Slattery T, et al: The receptor for advanced glycation end products (RAGE) is a cellular binding site for amphoterin. J Biol Chem 270: 25752-25761, 1995.
24. Yang H, Wang H, Czura CJ and Tracey KJ: The cytokine activity of HMGB1. J Leukoc Biol 78: 1-8, 2005.

25. Merenmies J, Pihlaskari R, Laitinen J, Wartiovaara J and Rauvala $\mathrm{H}$ : $30-\mathrm{kDa}$ heparin-binding protein of brain (amphoterin) involved in neurite outgrowth. Amino acid sequence and localization in the filopodia of the advancing plasma membrane. J Biol Chem 266: 16722-16729, 1991.

26. Taguchi A, Blood DC, del Toro G, et al: Blockade of RAGEamphoterin signalling suppresses tumour growth and metastases. Nature 405: 354-360, 2000

27. Park JS, Gamboni-Robertson F, He Q, et al: High mobility group box 1 protein interacts with multiple Toll-like receptors. Am J Physiol Cell Physiol 290: C917-C924, 2006.

28. Park JS, Arcaroli J, Yum HK, et al: Activation of gene expression in human neutrophils by high mobility group box 1 protein. Am J Physiol Cell Physiol 284: C870-C879, 2003.

29. Yu M, Wang H, Ding A, et al: HMGB1 signals through toll-like receptor (TLR) 4 and TLR2. Shock 26: 174-179, 2006.

30. Ivanov S, Dragoi AM, Wang X, et al: A novel role for HMGB1 in TLR9-mediated inflammatory responses to CpG-DNA. Blood 110: 1970-1981, 2007.

31. Tian J, Avalos AM, Mao SY, et al: Toll-like receptor 9-dependent activation by DNA-containing immune complexes is mediated by HMGB1 and RAGE. Nat Immunol 8: 487-496, 2007.

32. Oyama Y, Hashiguchi T, Taniguchi N, et al: High-mobility group box-1 protein promotes granulomatous nephritis in adenine-induced nephropathy. Lab Invest 90: 853-866, 2010.

33. Iwata Y, Furuichi K, Sakai N, et al: Dendritic cells contribute to autoimmune kidney injury in MRL-Faslpr mice. J Rheumatol 36: 306-314, 2009.

34. Zhou JG, Dong JY, Zhang LH and Wang J: Expression of high mobility group box chromosomal protein 1 in mice with lupus nephritis. Zhejiang Da Xue Xue Bao Yi Xue Ban 40: 200-206, 2011 (In Chinese).

35. Pisetsky DS: HMGB1: a smoking gun in lupus nephritis? Arthritis Res Ther 14: 112, 2012

36. Feng X, Hao J, Liu Q, et al: HMGB1 mediates IFN- $\gamma$-induced cell proliferation in MMC cells through regulation of cyclin D1/CDK4/p16 pathway. J Cell Biochem 113: 2009-2019, 2012.

37. Zickert A, Palmblad K, Sundelin B, et al: Renal expression and serum levels of high mobility group box 1 protein in lupus nephritis. Arthritis Res Ther 14: R36, 2012.

38. Bruchfeld A, Wendt M, Bratt J, et al: High-mobility group box-1 protein (HMGB1) is increased in antineutrophilic cytoplasmatic antibody (ANCA)-associated vasculitis with renal manifestations. Mol Med 17: 29-35, 2011

39. Kim J, Sohn E, Kim CS, Jo K and Kim JS: The role of highmobility group box-1 protein in the development of diabetic nephropathy. Am J Nephrol 33: 524-529, 2011.

40. Lin M, Yiu WH, Wu HJ, et al: Toll-like receptor 4 promotes tubular inflammation in diabetic nephropathy. J Am Soc Nephrol 23: 86-102, 2012

41. Penfold SA, Coughlan MT, Patel SK, et al: Circulating highmolecular-weight RAGE ligands activate pathways implicated in the development of diabetic nephropathy. Kidney Int 78: 287-295, 2010.

42. Torres VE, Harris PC and Pirson Y: Autosomal dominant polycystic kidney disease. Lancet 369: 1287-1301, 2007.

43. Nakamura T, Kawagoe Y, Ueda Y, Yamada S and Koide H: Hemoperfusion treatment in a septic shock patient with autosomal dominant polycystic kidney disease and increased HMGB1 protein levels. Blood Purif 32: 139-142, 2011.

44. Nakamura T, Sato E, Fujiwara N, et al: Changes in urinary albumin excretion, inflammatory and oxidative stress markers in ADPKD patients with hypertension. Am J Med Sci 343: 46-51, 2012.

45. Hu YM, Pai MH, Yeh CL, Hou YC and Yeh SL: Glutamine administration ameliorates sepsis-induced kidney injury by downregulating the high-mobility group box protein-1-mediated pathway in mice. Am J Physiol Renal Physiol 302: F150-F158, 2012.

46. Wang Q, Yao YM, Wang YB, et al: Effect of ethyl pyruvate on renal high mobility group box-1 protein expression and acute kidney injury in rats with delayed resuscitation after thermal injury. Zhonghua Wai Ke Za Zhi 45: 1210-1213, 2007 (In Chinese)

47. Chen J, Hartono JR, John R, et al: Early interleukin 6 production by leukocytes during ischemic acute kidney injury is regulated by TLR4. Kidney Int 80: 504-515, 2011 
48. Chung KY, Park JJ and Kim YS: The role of high-mobility group box-1 in renal ischemia and reperfusion injury and the effect of ethyl pyruvate. Transplant Proc 40: 2136-2138, 2008.

49. Wu H, Ma J, Wang P, et al: HMGB1 contributes to kidney ischemia reperfusion injury. J Am Soc Nephrol 21: 1878-1890, 2010.

50. Li J, Gong Q, Zhong S, et al: Neutralization of the extracellular HMGB1 released by ischaemic damaged renal cells protects against renal ischaemia-reperfusion injury. Nephrol Dial Transplant 26: 469-478, 2011.

51. Bao YS, Na SP, Zhang P, et al: Characterization of interleukin-33 and soluble ST2 in serum and their association with disease severity in patients with chronic kidney disease. J Clin Immunol 32: 587-594, 2012.

52. Bruchfeld A, Qureshi AR, Lindholm B, et al: High mobility group box protein-1 correlates with renal function in chronic kidney disease (CKD). Mol Med 14: 109-115, 2008.

53. Sato F, Maruyama S, Hayashi $\mathrm{H}$, et al: High mobility group box chromosomal protein 1 in patients with renal diseases. Nephron Clin Pract 108: c194-c201, 2008.

54. Leelahavanichkul A, Huang Y, Hu X, et al: Chronic kidney disease worsens sepsis and sepsis-induced acute kidney injury by releasing high mobility group box protein-1. Kidney Int 80 : $1198-1211,2011$.
55. Nakamura T, Sato E, Fujiwara N, et al: Positive association of serum levels of advanced glycation end products and high mobility group box-1 with asymmetric dimethylarginine in nondiabetic chronic kidney disease patients. Metabolism 58: 1624-1628, 2009.

56. D'Agati V and Schmidt AM: RAGE and the pathogenesis of chronic kidney disease. Nat Rev Nephrol 6: 352-360, 2010.

57. Lynch J, Nolan S, Slattery C, Feighery R, Ryan MP and McMorrow T: High-mobility group box protein 1: a novel mediator of inflammatory-induced renal epithelial-mesenchymal transition. Am J Nephrol 32: 590-602, 2010.

58. Sandim V, Pereira D, Ornellas A and Alves G: Renal cell carcinoma and proteomics. Urol Int 84: 373-377, 2010.

59. Lin L, Zhong K, Sun Z, Wu G and Ding G: Receptor for advanced glycation end products (RAGE) partially mediates HMGB1-ER Ks activation in clear cell renal cell carcinoma. J Cancer Res Clin Oncol 13: 11-22, 2012.

60. Ganji MR and Harririan A: Chronic allograft dysfunction: major contributing factors. Iran J Kidney Dis 6: 88-93, 2012.

61. Wang S, Schmaderer C, Kiss E, et al: Recipient Toll-like receptors contribute to chronic graft dysfunction by both MyD88- and TRIF-dependent signaling. Dis Model Mech 3: 92-103, 2010 